\title{
Hirsutism - Evaluation and Treatment
}

\author{
Nazma Akter ${ }^{1}$, Nazmul Kabir Qureshi ${ }^{2}$
}

\begin{abstract}
Hirsutism is a distressing clinical condition seen in adult female patients. The cause is mainly hyperandrogenism, which may be ovarian or adrenal. The most common pathological cause is usually polycystic ovary syndrome. It may be part of a rare metabolic syndrome, drug induced, or just idiopathic. It requires in depth clinical evaluation and investigation for treatment. Serum testosterone measurements are not needed in many cases. Hirsutism has a huge psychosocial impact, especially in the young females. This article reviews the current evaluation guidelines and management of hirsutism.
\end{abstract}

Keywords: Hirsutism; evaluation; treatment.

Delta Med Col J. Jan 2016;4(1):35-44

\section{Sources and selection criteria}

We reviewed all references to "Hirsutism" in Medline, the PudMed and selected randomized controlled trials for inclusion in the evidence base. We also referred to the Endocrine Society's clinical practice guidelines for the management of hirsutism, AACE medical guidelines for clinical practice for the diagnosis and treatment of hyperandrogenic disorders and The New England Journal of Medicine. Clinical experience is based on a tertiary referral endocrine service.

\section{Introduction}

Hirsutism is defined as excessive hair growth in women, occurring in anatomic areas where the hair follicles are most androgen sensitive. It manifests as an excessive or inappropriate development and growth of the pilosebaceous unit (PSU). ${ }^{1}$ It affects around $5-10 \%$ of women ${ }^{2,3}$ and is a common presenting complaint in the dermatological outpatient department (OPD) for cosmetic reasons. Generally Americans are less hairy than are Mediterranean people. About one in three non-Scandinavian or non-Asian women has some hair on the upper lip, periareolar and suprapubic areas. East Asians tend to be less hairy than Euro-Americans; with no difference in testosterone levels. ${ }^{4}$ Hormonal levels are also normal in prepubertal simple hypertrichosis ${ }^{5}$, which is the appropriate term for hairiness of non hormonal origin, in contrast to hirsutism and its extreme, virilization. Familial hairiness may be genetic or hormonal. Age-dependent variation in

1. Resident Physician, Dept. of Medicine, MARKS Medical College \& Hospital, Dhaka, Bangladesh.

2. Specialist, Dept. of Medicine, United Hospital Limited, Dhaka, Bangladesh.

Correspondence: Dr. Nazma Akter. e-mail: nazma_aktar_endo@yahoo.com 
hairiness has also been reported. ${ }^{6}$ The most important determinant in making the diagnosis is a change in the form and rate of hair growth. A technique has been developed to assess hirsutism with video equipment and computer software. It is not only imperative to identify the cause of hirsutism but also important to know how to recommend the right treatment based on the main causative factor. ${ }^{7}$

\section{Aetiology}

Classically, hirsutism has been considered a marker of increased androgen levels in females from increased production of androgens (i.e. testosterone) either by the adrenals or due to an ovarian disease. ${ }^{8,9}$ The ovarian causes for hyperandrogenism are polycystic ovarian syndrome (PCOS) and ovarian tumours. Adrenal causes include Cushing's syndrome, androgen-producing tumours, and congenital adrenal hyperplasia (CAH), most commonly due to 21-hydroxylase deficiency. Less common causes include the hyperandrogenic-insulin resistant-acanthosis nigricans syndrome (HAIRAN). Hyperprolactinemia by increasing adrenal dihydroepiandrosterone sulfate (DHEA-S) production may cause hirsutism. Androgenic drugs are also an important cause of hirsutism, e.g., oral contraceptives, danazol, metoclopramide, anabolic steroid, methyldopa, phenothiazines. ${ }^{10}$ About $20 \%$ of the patients may present with idiopathic hirsutism (IH) with normal androgen levels and ovarian function. The cause of increased hair in these women is thought to be related to disorders in peripheral androgen activity. ${ }^{11}$ Onset of IH occurs shortly after puberty with slow progression. Polycystic ovarian syndrome and IH account for $90 \%$ of the hirstutism in women. Hirsutism can also occur in some premenopausal women and continue for a few years after menopause. This is due to decrease in ovarian estrogen secretion with continuous androgen production. ${ }^{12}$

\section{Pathogenesis}

Androgens cause transformation of vellus (fine, soft, unpigmented) hair to terminal hair in androgen dependent areas of hair growth. ${ }^{1}$ Before puberty, hair is vellus (small, straight, and fair), and the sebaceous glands in androgen-sensitive follicles are small. In response to the increased levels of androgens at puberty, vellus follicles in specific areas develop into terminal hairs (larger, curlier, and darker, hence more visible), becoming sexual-hair follicles. Higher androgen levels are required for the growth of beard than for the growth of pubic and axillary hair. In other areas (e.g., the forehead and cheeks), the increased androgen levels dramatically increase the size of the sebaceous glands, but the hair remains vellus; the reason for this differential response is unclear. ${ }^{13,14}$ Hirsutism is attributed either to increased production or increased sensitivity of the hair follicles to circulating androgen (testosterone). 8,9 Most women with androgen levels that are twice the upper limit of the normal range or higher have some degree of hirsutism. ${ }^{15}$ Majority of testosterone is secreted either by the ovaries or adrenals (80\%). A small amount of circulating testosterone is derived from the conversion of androgenic precursors, mainly androstenedione (derived from the ovaries and adrenals) and dihydroepiandrosterone (DHEA derived from the adrenals) in liver, skin and adipose tissue. However, only $1-2 \%$ of testosterone is in free form and is the active androgen. About $98-99 \%$ is bound to steroid hormone binding globulin (SHBG), cortisol binding globulin, or nonspecifically to albumin and other proteins and is biologically inactive. Only free testosterone is converted to dihydrotestosterone (DHT), by the enzyme 5-alpha reductase type 2 isoenzyme present in the outer root sheath of the hair follicles. This isoenzyme predominates in the testes, prostate, and the hair follicles of beard and genital hair., 8,9 Some women with excess androgen have no skin manifestations or they may have seborrhea, acne, or alopecia without hirsutism. In other women hirsutism develops without the presence of excess androgen (termed idiopathic hirsutism). ${ }^{15}$ Free testosterone seems to be the main bioactive portion of plasma testosterone. ${ }^{16,17}$ The level of free 
testosterone is often elevated when the total testosterone level is normal in hirsute women. This reflects the relatively low levels of sex hormone-binding globulin in such women, which determines the fraction of plasma testosterone that is free or bound to albumin, ${ }^{18}$ so that the total testosterone level may be normal despite excess androgen levels. ${ }^{19}$

\section{Clinical Features}

Hirsute women usually present with increased growth of terminal hair at sides of the face, upper lip, chin, upper back, shoulders, sternum, and upper abdomen. Ferriman and Gallwey ${ }^{3}$ devised a scoring system for clinical quantification of hirsutism (Table I). In their study of 161 women aged 18 to 38 years, they graded density of terminal hair at nine different body sites under androgen effect from 0 (absence of terminal hairs) through 4 (extensive terminal hair growth) and concluded that hirsutism was represented by a score of 8 or more. Approximately half of women with mild hirsutism (i.e., hirsutism with a score of 8 to 15 , out of a maximum of 36 , on the Ferriman-Gallwey scale) have the idiopathic condition, ${ }^{15}$ whereas in the remainder of those women and in most of those with more marked hirsutism, androgen levels were elevated. Hyperandrogenism is most often caused by the PCOS. ${ }^{20,21}$ However, this is a subjective scale and hence not universally adopted. ${ }^{3}$

\section{Diagnostic Evaluation of Hirsute Patients} When a female patient presents with the chief complaint of increased growth of hair, it is imperative to see whether the coarse hairs are localized in male distribution or there is generalized increase in growth of the hair on all of the body (hypertrichosis). ${ }^{22,23}$ After determining hirsutism, the following steps can be undertaken to find the cause ${ }^{24}$ (Fig. 1). A thorough history and physical examination provide the most important initial diagnostic information, whereas laboratory tests should usually serve to confirm the presence of hyperandrogenemia.
Table I: Definition of Hair Grading at Each of 19 Sites (Ferriman-Gallwey Scale) ${ }^{3}$

\begin{tabular}{|c|c|c|}
\hline Site & Grade & Definition \\
\hline \multirow[t]{4}{*}{ Upper lip } & 1 & A few hairs at outer margin \\
\hline & 2 & A small mustache at outer margin \\
\hline & 3 & A mustache extending halfway from outer margin \\
\hline & 4 & A mustache extending to midline \\
\hline \multirow[t]{3}{*}{ Chin } & 1 & A few scattered hairs \\
\hline & 2 & Scattered hairs with small concentrations \\
\hline & $3 \& 4$ & Complete cover, light and heavy, respectively \\
\hline \multirow[t]{4}{*}{ Sideburns } & 1 & Few non terminal hairs \\
\hline & 2 & More non terminal hairs \\
\hline & 3 & Terminal hair on side of face \\
\hline & 4 & Terminal hair extending to mandible \\
\hline \multirow[t]{3}{*}{ Neck } & 1 & Few hairs on neck \\
\hline & 2 & More hairs on neck \\
\hline & $3 \& 4$ & Complete cover, light and heavy respectively \\
\hline \multirow[t]{2}{*}{ Chest } & 1 & Circumareolar hairs \\
\hline & 2 & With midline hair in addition \\
\hline \multicolumn{3}{|l|}{ Upper back } \\
\hline & 3 & Fusion of these areas, with three-quarter cover \\
\hline & 4 & Complete cover \\
\hline \multirow[t]{3}{*}{ Lower back } & 1 & A few scattered hairs \\
\hline & 2 & Rather more, still scattered \\
\hline & $3 \& 4$ & Complete cover, light and heavy, respectively \\
\hline \multirow[t]{2}{*}{ Buttocks } & 1 & A sacral tuft of hair \\
\hline & 2 & With some lateral extension \\
\hline \multirow[t]{3}{*}{ Upper abdomen } & $3 \& 4$ & Three-quarter cover or complete cover, respectively \\
\hline & $1 \& 2$ & Few or many hairs, respectively, over lower buttocks \\
\hline & $3 \& 4$ & Hair extending to upper buttocks, light and heavy, respectively \\
\hline \multirow[t]{4}{*}{ Lower abdomen } & 1 & A few midline hairs \\
\hline & 2 & Rather more, still midline involvement \\
\hline & $3 \& 4$ & Half and full cover, respectively \\
\hline & 1 & A few midline hairs \\
\hline \multirow[t]{2}{*}{ Inguinal area } & 2 & A midline streak of hair \\
\hline & 3 & A midline band of hair \\
\hline \multirow[t]{3}{*}{ Perianal area } & 4 & An inverted V-shaped growth \\
\hline & 1 & Pubic hair extending to inguinal area \\
\hline & 2 & A few hairs below inguinal area \\
\hline \multirow[t]{3}{*}{ Arm } & 1 & Hair encircling introitus and anus \\
\hline & 2 & Hair extending to inner thigh \\
\hline & $3 \& 4$ & Hair on inner thigh and buttocks, light and heavy, respectively \\
\hline Forearm & 1 & Sparse growth affecting no more than a quarter of the limb surface \\
\hline Thigh & 2 & More than this; cover still incomplete \\
\hline Leg & $3 \& 4$ & Complete cover, light and heavy, respectively \\
\hline \multirow[t]{3}{*}{ Foot } & $1,2,3,4$ & As for arm \\
\hline & $1,2,3,4$ & As for arm \\
\hline & $1,2,3,4$ & As for arm \\
\hline \multirow[t]{2}{*}{ Toes } & 1 & A few hairs on dorsum of foot \\
\hline & 2 & More hair on dorsum of foot \\
\hline \multirow[t]{7}{*}{ Fingers } & $3 \& 4$ & Hair over one-half or three-quarters or more, respectively, of dorsum \\
\hline & $1 \& 2$ & Few hairs or many hairs, respectively, on big toe \\
\hline & $3 \& 4$ & Few hairs or many hairs, respectively, on other toes \\
\hline & 1 & Few hairs on proximal phalanx-dorsal surface \\
\hline & 2 & Many hairs on proximal phalanx-dorsal surface \\
\hline & 3 & Few hairs on 2nd phalanx-dorsal surface \\
\hline & 4 & Many hairs on 2nd phalanx-dorsal surface \\
\hline
\end{tabular}




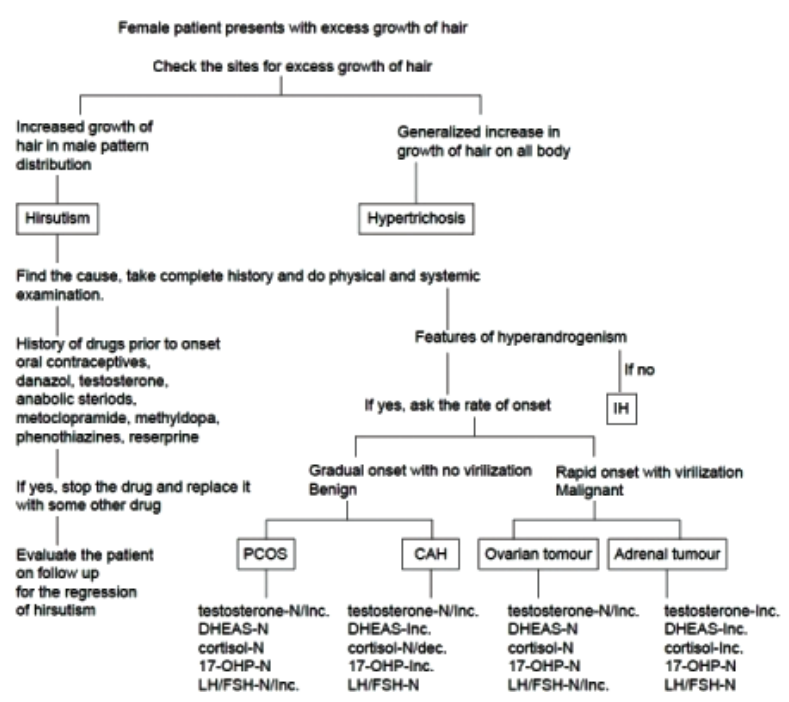

Fig. 1: Algorithm showing evaluation of a female patient with excess growth of hair [IHIdiopathic hirsutism, CAH- Congenital adrenal hyperplasia, PCOS- Polycystic ovarian syndrome, DHEAS- Dehydroepiandrosterone sulfate, 17 OHP- 17 Hydroxy progesterone, LHLuteinizing Hormone, FSH- Follicle stimulating Hormone, (+) present, (-) absent, N- Normal, Inc.- Increased, dec.- Decreased]

\section{History and physical examination}

A) The history should include information about the patient's age at thelarche, adrenarche, and menarche. Detailed history including age of onset (puberty, middle age, menopause), rate of onset of symptoms (gradual or sudden), any signs or symptoms of virilisation (acne, deepening of voice, infrequent menstruation, loss of breast tissue or loss of normal female body contour, clitoromegaly, increased libido, increased muscle mass as in shoulder girdle, malodorous perspiration, etc.), history of weight gain or diabetes should be taken. One should also note medications used and their effects on acne and hirsutism. ${ }^{24}$ If obesity is present, the time of onset and the progression should be noted. The character of the menstrual cycles (their frequency, duration and occurrence of dysmenorrhea) along with the reproductive history, including miscarriages, should be evaluated. The family history is also important in determining whether other family members have hirsutism, acne, infertility, diabetes mellitus and cardiovascular disease. ${ }^{25,26}$

B) Complete general physical and systemic examination should be done including palpation of abdomen for any ovarian mass. The uterus should be examined for size and the presence of tumors. In addition to the usual vital signs, the measurements of height, weight, and waist circumference and the determination of the body mass index and the waist-to-hip ratio (WHR) are essential. ${ }^{27}$ The WHR (normal women, $<0.8$ ) and body mass index are important in assessing the degree of obesity in women with hyperandrogenism. ${ }^{28}$ Particular attention should be paid to the degree and distribution of cutaneous manifestations of androgen excess (hirsutism, acne, and alopecia). The degree of hirsutism can best be documented and graded by using a system such as the one presented in Table 1. In addition, the presence of clitoral hypertrophy, acanthosis nigricans and examination of the thyroid gland and breasts (presence of galactorrhea) should be emphasized. ${ }^{29}$

\section{Laboratory evaluation}

If drug is the cause, simple withdrawal of the drug should be helpful. For all other cases, laboratory evaluation of the serum markers to know the exact etiology should be done. The various serum markers are -

a) Testosterone - According to Endocrine Society clinical practice guidelines, $2008^{30}$ they suggest against testing for elevated androgen levels in women with isolated mild hirsutism because the likelihood of identifying a medical disorder that would change management or outcome is low. But they suggest testing for elevated androgen levels in women with:

- Moderate or severe hirsutism.

- Hirsutism of any degree when it is sudden in onset, rapidly progressive, or when associated with any of the following:

- menstrual irregularity or infertility.

- central obesity.

- acanthosis nigricans. 
- rapid progression.

- clitoromegaly.

Determinations of androgen levels are most accurately performed by a specialty laboratory. ${ }^{31}$ The normal upper limit for total plasma testosterone levels in women varies from about 70 to $90 \mathrm{ng} / \mathrm{dL}$ ( 2.43 to $3.12 \mathrm{nmol} / \mathrm{L})$. This is because there are systematic differences among assays, ${ }^{32}$ and many laboratories provide excessively broad normal ranges because the general population includes women with unrecognized androgen excess. ${ }^{33,34}$

Serum testosterone may be normal to increased in case of benign pathology as PCOS and CAH but would be definitely raised $(>200 \mathrm{ng} / \mathrm{mL})$ in case of malignant tumour of the adrenal or ovary. ${ }^{24}$

b) Dehydroepiandrosterone sulfate (DHEAS) Routine testing for other androgens is of little use. ${ }^{15,20,35}$ The level of dehydroepiandrosterone sulfate is increased in approximately $15 \%$ of women who have normal levels of total and free testosterone. A mildly elevated level in a woman with a normal free testosterone level is unlikely to be clinically relevant aside from being associated with acne.14,36,37 Raised DHEAS (>700 $\mu \mathrm{g} / \mathrm{dL})$ always indicates an adrenal cause, benign or malignant. ${ }^{24}$

c) 17 Hydroxy progesterone - This serum marker is unique for congenital adrenal hyperplasia. The measurement should be done between 0700 and 0900 hours in the early follicular phase of the menstrual cycle. Levels less than $200 \mathrm{ng} / \mathrm{dL}$ excludes the disease. Mildly increased levels between 300 and 1,000 ng/dL require an $\mathrm{ACTH}$ stimulation test. Cosyntropin (synthetic ACTH), $250 \mu \mathrm{g}$, is administered intravenously, and levels of 17-hydroxyprogesterone are measured before and one hour after the injection. Post-stimulation value of $>1,000 \mathrm{ng} / \mathrm{dL}$ constitutes a positive test. ${ }^{24}$

d) Twenty four hour urine free cortisol should be measured in women with signs and symptoms of Cushing's syndrome.

e) LH/FSH greater than 3 is indicative of PCOS. ${ }^{38}$

f) Prolactin would be raised in hyperprolactinemia due to hypothalamic disease or a pituitary tumour. g) Serum TSH: Hypophyseal hypothyroidism ${ }^{39}$ can act as a cofactor in hirsutism causing raised TSH.

3) Pelvic ultrasonography can be done to detect an ovarian neoplasm or a polycystic ovary.

4) Magnetic resonance imaging (MRI) or computed tomography (CT) of the adrenal region is useful for diagnosis. ${ }^{24}$

\section{Management}

Hirsutism can be reduced with the use of cosmetic and hormonal therapy for as long as treatment is given. Moderate, permanent reduction of hirsutism can often be achieved by physical means in optimal circumstances.

\section{Cosmetic and Physical Measures}

Cosmetic measures are the cornerstone of care for hirsutism. ${ }^{40}$ Most women resort to removal of hair by different depilating methods, such as plucking, shaving, and waxing before presenting to the clinic. Though simple and inexpensive, these methods are temporary and have their own side effects like physical discomfort, scarring, folliculitis, irritant dermatitis or discoloration. Electrolysis has also been used for the removal of the hair. With repeated treatments, the efficacy ranges from 15 to $50 \%$ permanent hair loss. ${ }^{41}$ However, it is difficult to treat large areas like hairs on the chest or upper back with electrolysis and it can be time consuming. The Food and Drug Administration has permitted the marketing of many laser devices, as well as equivalent flash lamps, for permanent hair reduction. Wave lengths of between 694 and $1064 \mathrm{~nm}$ damage hair follicles through the combination of relatively selective heat absorption by dark hairs and penetration of the wave lengths into the dermis. Light-skinned women are the best candidates, since they require lower energy pulses than women with dark skin. Those with heavily tanned or darker skin require the use of lasers with built-in cooling devices and adjustment of energy levels to minimize the risk of dermatologic side effects. Laser treatment covers a somewhat wider surface area, with fewer side effects and less pain, than electrolysis, temporarily reduces by a factor of about four the need for simple cosmetic measures for several months after a single session, and permanently reduces hair 
density by 30 percent or more with three to four treatments at a site. Electrolysis involves the insertion of an electrode to destroy individual follicles. Both laser therapy and electrolysis require delivery by trained personnel, are repetitious, expensive, and painful, are practical only for the treatment of limited areas, and may result in local reactions, including burns, dyspigmentation, and scarring. Laser hair removal is most suitable for idiopathic hirsutism with the normal androgen levels. ${ }^{42,43}$

\section{Treatment with drugs}

Before starting medicine, diet and exercise should be advised to all women with PCOS. For all obese women, weight loss as a therapy should be advised. Upper body obesity has been shown to be associated with a reduced sex hormone-binding globulin level and increased free testosterone levels in both non-hirsute and hirsute women and can contribute to hirsutism. Drugs are indicated for treatment when hyperandrogenism is confirmed by various laboratory tests. The following drugs can be used:

Hormonal therapies act by either suppressing androgen production or blocking the action of androgens within the skin. The suppression or blockage of androgen causes hairs to revert toward the prepubertal vellus type. The maximal effect requires 9 to 12 months of treatment because of the long duration of the hair-growth cycle. ${ }^{13}$

\section{Estrogen-Progestin Oral Contraceptives}

Oral contraceptives (OCPs) suppress plasma-testosterone levels, particularly the level of free testosterone, mainly by inhibiting ovarian function. ${ }^{13}$ This method of treatment can reduce by half the need for shaving ${ }^{4}$ and can arrest the progression of hirsutism from various causes, but it will not reverse hirsutism ${ }^{45,46}$; cosmetic measures should also be used. Oral contraceptive therapy reduces hyperandrogenism via a number of mechanisms including suppression of $\mathrm{LH}$ secretion (and therefore ovarian androgen secretion), stimulation of hepatic production of sex hormone binding globulin (SHGB), thereby increasing androgen binding in serum and reducing serum free androgen concentrations, a slight reduction in adrenal androgen secretion, and a slight blockage in the binding of androgens to their receptor. OCPs provide the additional benefits of bleeding control and contraception. ${ }^{47}$

\section{Androgen receptor blockers}

For the substantial reduction of hirsutism, antiandrogens are required. Competitive inhibitors of androgen binding to the androgen receptor are superior to drugs that interfere with testosterone metabolism. ${ }^{48-51}$ They are effective regardless of the cause of hyperandrogenism and may be helpful in the treatment of idiopathic hirsutism. ${ }^{45}$

1) Spironolactone (SPA), an aldosterone antagonist, exhibits dose-dependent competitive inhibition of the androgen receptor. ${ }^{52}$ SPA also has variable progestational activity and decreases production of ovarian androgens. It has an inhibitory effect on $5 \alpha$-reductase activity ( $5 \alpha-$ RA) and competes with androgens for binding to SHBG. The starting dose is $50 \mathrm{mg}$ twice daily and may be increased to a total daily dose of $200 \mathrm{mg}$. It takes at least six months to have any beneficial effect. The use of SPA is recommended with the OCP which provides adequate contraception and also helps to minimize the dysfunctional uterine bleeding. ${ }^{53}$ Side effects include polyuria and hypotension with associated headaches, fatigue, or even syncope. SPA should not be used in conjunction with other potassium-sparing diuretics, thiazides, in renal insufficiency, or with excess potassium intake, since patients may develop life-threatening hyperkalemia. It is recommended that serum electrolytes and blood pressure be evaluated two to four weeks after treatment is started. Other minor side effects commonly associated with SPA use include gastritis/dyspepsia and dry skin; SPA should be taken with food as this increase its absorption and reduces its potential for gastritis. ${ }^{54}$ Absolute contraindications to SPA use include renal insufficiency, anuria, chronic renal impairment, hyperkalemia, pregnancy and abnormal uterine bleeding. 
2) Cyproterone Acetate (CPA), has strong progestogenic and antiandrogen properties. It produces a decrease in circulating testosterone and androstenedione levels through a reduction in circulating LH and has been used as an effective treatment for hirsutism. ${ }^{50} \mathrm{CPA}$ is available in combination with ethinyl estradiol (EE) $(2 \mathrm{mg}$ CPA and $35 \mu \mathrm{g}$ EE/tablet).

The use of either spironolactone or cyproterone acetate can be expected to reduce the Ferriman-Gallwey score by 15 to 40 percent within 6 months after the start of therapy, although there is considerable variation among individual women, with the maximum effect at 9 to 12 months. ${ }^{2}$

\section{5- $\alpha$ reuctase inhibitors (ARI)}

Finasteride, a 5- $\alpha$ reductase inhibitor (ARI) has been found to be effective in the treatment of IH. Finasteride primarily inhibits type $25 \alpha$-reductase activity. ${ }^{55}$ It is less effective for hirsutism than are anti androgens. ${ }^{51}$ All these $5 \alpha$ - reductase inhibitors agents have the potential of feminizing a male fetus. Hence, effective contraception must be used by patients on these drugs.

\section{Gonadotrophin-releasing hormone (GnRH agonists)}

Consideration of GnRH agonist (GnRHa) therapy is based on the assumption that hirsutism is at least in part gonadotropin-dependent. The action of chronic GnRHa therapy is to inhibit luteinizing hormone (LH) and to a lesser extent follicle-stimulating hormone secretion, thereby leading to a decline in ovarian function and consequently decreased ovarian androgen production. $^{46}$ This therapy is parenteral and reserved for women with severe hirsutism who don't respond to the OCPs and antiandrogens. Long-acting GnRH analogues decrease gonadotrophin secretion and therefore reduce ovarian stimulation and hence testosterone. Estrogen production is also reduced. Hence, therapy is usually used in combination with an oral contraceptive pill containing estrogen and progestin. 56

\section{Other drugs}

- Glucocorticoid therapy (typically, 5 to $7.5 \mathrm{mg}$ of prednisone at bedtime) may improve hirsutism in patients with nonclassic congenital adrenal hyperplasia. However, the effect of glucocorticoids on hirsutism due to other causes is unclear, and slight overdosing, as can occur even at recommended doses, is associated with serious side effects. ${ }^{46}$

- In women with polycystic ovary syndrome, insulin sensitizers (metformin or thiazolidinediones) promote ovulation and lower androgen levels by about 20 percent, but there is little evidence of a clinically significant improvement in hirsutism with the use of these agents. ${ }^{58,59}$

\section{Biological modifiers of hair follicular growth}

Eflornithine hydrochloride is a new agent, which is used as a topical cream (13.9\%) for decreasing or arresting facial hair growth in women. It is thought to inhibit hair growth by inhibiting an enzyme involved in keratin synthesis. It is a potent, irreversible inhibitor of the enzyme ornithine decarboxylase, which is necessary for production of the polyamines that mediate cell migration, proliferation, and differentiation. ${ }^{60}$ Binding of DHT to the androgen receptor is associated with stimulation of ornithine decarboxylase synthesis and proliferation of hair matrix cells. The cream is applied to the face twice a day. Gradual improvement is seen in six to eight weeks. It can also be used in combination with laser treatments for better effects. ${ }^{61}$

\section{Conclusion}

Hirustism requires in depth clinical evaluation and investigation for treatment. For women presenting with hirsutism, the medical history and physical examination should assess whether there are any features to suggest the presence of a neoplasm or endocrinopathy, particularly polycystic ovary syndrome. Approaches to laboratory testing vary among specialists. For pharmacological therapy, oral contraceptives are recommended for the majority of women. Adding an anti androgen may 
be needed after six months if the response is suboptimal. Anti androgen monotherapy is not recommended unless adequate contraception is used. For women who choose hair removal therapy, photo eplilation with lasers is the preferred choice. For all pharmacologic therapies for hirsutism, a trial of at least 6 months should be continue before making changes in dose or adding medication.

\section{References}

1. Steinberger E, Rodriguez-Rigau LJ, Smith KD, Held B. The Menstrual Cycle and Plasma Testosterone Levels in Women with Acne. J Am Acad Dermatol. 1981;4:54-58.

2. Mcknight E. The Prevalence of "Hirsutism" in Young Women. Lancet. 1964;1:410-13.

3. Ferriman D, Gallwey JD. Clinical Assessment of Body Hair Growth in Women. J Clin Endocrinol Metab. 1961;21:1440-47.

4. Ewing JA, Rouse BA. Hirsutism, Race and Testosterone Levels: Comparison of East Asians and Euroamericans. Hum Biol. 1978;50:209-15.

5. Balducci R, Toscano V. Bioactive and Peripheral Androgens in Prepubertal Simple Hypertrichosis. Clin Endocrinol (Oxf). 1990;33:407-14.

6. Lunde O. A Study of Body Hair Density and Distribution in Normal Women. Am J Phys Anthropol. 1984;64:179-84.

7. Gruber DM, Berger UE, Sator MO, Horak F, Huber JC. Computerized Assessment of Facial Hair Growth. Fertil Steril. 1999;72:737-39.

8. Rittmaster RS. Hirsutism [review]. Lancet. 1997;349:19-95.

9. Rosenfield RL. Clinical Practice. Hirsutism. N Engl J Med. 2005;353:2578-88.

10. Leung AK, Robson WL. Hirsutism. Int J Dermatol. 1993;32:773-77.

11. Azziz R, Carmina E, Sawaya ME. Idiopathic Hirsutism. Endocr Rev. 2000;21:347-62.

12. Martin KA, Chang RJ, Ehrmann DA, Ibanez L, Lobo RA, Rosenfield RL, et al. Evaluation and Treatment of Hirsutism in Premenopausal Women: An Endocrine Society Clinical Practice Guideline. J Clin Endocrinol Metab. 2008;93:1105-20.
13. Deplewski D, Rosenfield RL. Role of Hormones in Pilosebaceous Unit Development. Endocr Rev. 2000;21:363-92.

14. Rosenfield RL. Hirsutism and the Variable Response of the Pilosebaceous Unit to Androgen. J Invest Dermatol. 2005;10(3):205-208.

15. Reingold SB, Rosenfield RL. The Relationship of Mild Hirsutism or Acne in Women to Androgens. Arch Dermatol. 1987;123:209-12.

16. Rosenfield RL, Moll GW Jr. The Role of Proteins in the Distribution of Plasma Androgens and Estradiol. In: Molinatti GM, Martini L, James VHT, editors. Androgenization in Women: Pathophysiology and Clinical Concepts. New York: Raven Press; 1983. p.25-45.

17. Rosner W. The Functions of Corticosteroid-Binding Globulin and Sex Hormone Binding Globulin: Recent Advances. Endocr Rev. 1990;11:80-91.

18. Moll GW Jr, Rosenfield RL. Testosterone Binding and Free Plasma Androgen Concentrations under Physiologic Conditions: Characterization by Flow Dialysis Technique. J Clin Endocrinol Metab. 1979;49:730-36.

19. Hogeveen KN, Cousin P, Pugeat M, Dewailly D, Soudan B, Hammond GL. Human Sex Hormone-Binding Globulin Variants Associated with Hyperandrogenism and Ovarian Dysfunction. J Clin Invest. 2002;109:973-81.

20. Azziz R, Sanchez LA, Knochenhauer ES, Moran C, Lazevby J, Stephen KC, et al. Androgen Excess in Women: Experience with Over 1000 Consecutive Patients. J Clin Endocrinol Metab. 2004; 89:453-62.

21. Ehrmann DA, Rosenfield RL, Barnes RB, Brigell DF, Sheikh Z. Detection of Functional Ovarian Hyperandrogenism in Women With Androgen Excess. N Engl J Med. 1992;327:157-62.

22. Gilchrist VJ, Hecht BR. A Practical Approach to Hirsutism. Am Fam Physician. 1995;52:1837-46.

23. Hunter MH, Carek PJ. Evaluation and Treatment of Women with Hirsutism. Am Fam Physician. 2003;67:65-72.

24. Lin-Su K, Nimkarn S, New MI. Congenital Adrenal Hyperplasia in Adolescents: Diagnosis and Management. Ann N Y Acad Sci. 2008;1135:95-98. 
25. Steinberger E, Ayala C, Hsi B, Smith KD, Rodriguez-Rigau LJ, Weidman R, et al. Utilization of Commercial Laboratory Results in Management of Hyperandrogenism in Women. Endocr Pract. 1998;4:1-10.

26. Ayala C, Steinberger E, Smith KD, Rodriguez-Rigau LJ, Petak SM. Serum Testosterone Levels and Reference Ranges in Reproductive-Age Women. Endocr Pract. 1999;5:322-29.

27. AACE/ACE Obesity Task Force. AACE/ACE Position Statement on the Prevention, Diagnosis, and Treatment of Obesity (1998 Revision). Endocr Pract. 1998;4:297-350.

28. Prineas RJ, Folsom AR, Kaye SA. Central Adiposity and Increased Risk of Coronary Artery Disease Mortality in Older Women. Ann Epidemiol. $1993 ; 3: 35-41$.

29. Polson DW, Adams J, Wadsworth J, Franks S. Polycystic Ovaries - A Common Finding in Normal Women. Lancet. 1988;1:870-72.

30. Martin KA, Chang RJ, Ehrmann DA, Ibanez L, Lobo RA, Rosenfield RL, et al. Evaluation and Treatment of Hirsutism in Premenopausal Women: An Endocrine Society Clinical Practice Guideline. The Journal of Clinical Endocrinology \& Metabolism. 2008;93(4):1105-20.

31. Goodman NF, Bledsoe MB, Cobin RH, Futterweit W, Goldzieher JW, Petak SM, et al. American Association of Clinical Endocrinologists Medical Guidelines for Clinical Practice for the Diagnosis and Treatment of Hyperandrogenic Disorders. Endocr Pract. 2001;7(2):120-34.

32. Taieb J, Mathian B, Millot F, Patricot MC, Mathieu E, Queyrel N, et al. Testosterone Measured by 10 Immunoassays and by Isotope-Dilution Gas Chromatography-Mass Spectrometry in Sera From 116 Men, Women, and Children. Clin Chem. 2003;49:1381-95.

33. Polson DW, Adams J, Wadsworth J, Franks S. Polycystic Ovaries - A Common Finding in Normal Women. Lancet. 1988;1:870-72.

34. Legro RS, Driscoll D, Strauss JF III, Fox J, Dunaif A. Evidence for a Genetic Basis for Hyperandrogenemia in Polycystic Ovary Syndrome. Proc Natl Acad Sci USA. 1998;95:14956-60.
35. Wild RA, Umstot ES, Andersen RN, Ranney GB, Givens JR. Androgen Parameters and Their Correlation with Body Weight in One Hundred Thirty-Eight Women Thought to Have Hyperandrogenism. Am J Obstet Gynecol. 1983;146:602-606.

36. Marynick SP, Chakmakjian ZH, McCaffree DL, Herndon JH Jr. Androgen Excess in Cystic Acne. N Engl J Med. 1983;308:981-86.

37. Azziz R, Dewailly D, Owerbach D. Nonclassic Adrenal Hyperplasia: Current Concepts. J Clin Endocrinol Metab. 1994;78:810-15.

38. Chang RJ, Katz SE. Diagnosis of Polycystic Ovary Syndrome. Endocrinol Metab Clin North Am. 1999;28:397-408.

39. Schmidt JB, Lindmaier A, Spona J. Hyperprolactinemia and Hypophyseal Hypothyroidism as Cofactors in Hirsutism and Androgen-Induced Alopecia in Women. Hautarzt. 1991;42:168-72.

40. Azziz R. The Evaluation and Management of Hirsutism. Obstet Gynecol. 2003;101:995-1007.

41. Wagner RF Jr. Physical Methods for the Management of Hirsutism. Cutis. 1990;45:19-26.

42. Dierickx CC. Hair Removal by Lasers and Intense Pulsed Light Sources. Dermatol Clin. 2002;20:135-46.

43. Battle EF Jr, Hobbs LM. Laser-assisted Hair Removal for Darker Skin Types. Dermatol Ther. 2004; 17:177-83.

44. Hancock KW, Levell MJ. The Use of Estrogen-Progestogen Preparations in the Treatment of Hirsutism in the Female. J Obstet Gynaecol Br Commonw. 1974;81:804-11.

45. Azziz R, Carmina E, Sawaya ME. Idiopathic Hirsutism. Endocr Rev. 2000;21:347-62.

46. Heiner JS, Greendale GA, Kawakami AK, Lapolt PS, Fisher M, Young D, et al. Comparison of a Gonadotropin-Releasing Hormone Agonist and a Low Dose Oral Contraceptive Given Alone or Together in the Treatment of Hirsutism. J Clin Endocrinol Metab. 1995;80:3412-18.

47. Fitzgerald C, Elstein M, Spona J. Effect of Age on the Response of the Hypothalamo-Pituitary-Ovarian Axis to a Combined Oral Contraceptive. Fertil Steril. 1999;71:1079-84. 
48. Venturoli S, Marescalchi O, Colombo FM, Macrelli S, Ravaioli B, Bagnoli A, et al. A Prospective Randomized Trial Comparing Low Dose Flutamide, Finasteride, Ketoconazole, and Cyproterone Acetate- Estrogen Regimens in the Treatment of Hirsutism. J Clin Endocrinol Metab. 1999;84:1304-10.

49. Moghetti P, Tosi F, Tosti A, Negri C, Misciali C, Perrone F, et al. Comparison of Spironolactone, Flutamide, and Finasteride Efficacy in the Treatment of Hirsutism: A Randomized, Double Blind, Placebocontrolled Trial. J Clin Endocrinol Metab. 2000;85:89-94.

50. Van der Spuy ZM, le Roux PA. Cyproterone acetate for hirsutism. Cochrane Database Syst Rev. 2003;4:CD001125.

51. Farquhar C, Lee O, Toomath R, Jepson R. Spironolactone Versus Placebo or in Combination with Steroids for Hirsutism and/or Acne. Cochrane Database Syst Rev. 2003;4:CD000194.

52. Lobo RA, Shoupe D, Serafini P, Brinton D, Horton R. The Effects of Two Doses of Spironolactone on Serum Androgens and Anagen Hair in Hirsute Women. Fertil Steril. 1985;43:200-205.

53. Shaw JC. Spironolactone in Dermatological Therapy. J Am Acad Dermatol. 1991;24:236-43.

54. Spritzer PM, Lisboa KO, Mattiello S, Lhullier F. Spironolactone as a Single Agent for Long-Term Therapy of Hirsute Patients. Clin Endocrinol (Oxf). 2000;52:587-94.

55. Faloia E, Filipponi S, Mancini V, Di Marco S, M antero F. Effect of Finasteride in Idiopathic Hirsutism. J Endocrinol Invest. 1998;21:694-98.

56. de Leo V, Fulghesu AM, la Marca A, Morgante G, Pasqui L, Talluri B, et al. Hormonal and Clinical Effects of GnRH Agonist Alone, or in Combination with a Combined Oral Contraceptive or Flutamide in Women with Severe Hirsutism. Gynecol Endocrinol. 2000;14:411-16.

57. Rittmaster RS, Givner ML. Effect of Daily and Alternate Day Low Dose Prednisone on Serum Cortisol and Adrenal Androgens in Hirsute Women. J Clin Endocrinol Metab. 1988;67:400-403.

58. Harborne L, Fleming R, Lyall H, Norman J, Sattar N. Descriptive Review of the Evidence for the Use of Metformin in Polycystic Ovary Syndrome. Lancet. 2003;361:1894-901.

59. Lord JM, Flight IH, Norman RJ. Insulinsensitising Drugs (Metformin, Troglitazone, Rosiglitazone, Pioglitazone, D-Chiro-Inositol) for Polycystic Ovary Syndrome. Cochrane Database Syst Rev. 2003;3:CD003053.

60. Malhotra B, Noveck R, Behr D, Palmisano M. Percutaneous Absorption and Pharmacokinetics of Eflornithine $\mathrm{HCl} 13 \%$ Cream in Women with Unwanted Facial Hair. J Clin Pharmacol. 2001;41:972-78.

61. Smith SR, Piacquadio DJ, Beger B, Littler C. Eflornithine Cream Combined with Laser Therapy in the Management of Unwanted Facial Hair Growth in Women: A Randomized Trial. Dermatol Surg. 2006;32:1237-43 